Original Research Paper

\title{
A New Experimental Method for Generating Locus of Control using a Wooden Labyrinth
}

\author{
${ }^{1}$ Katherine E. Riley, ${ }^{2}$ Maureen A. Sullivan and ${ }^{1}$ Charles I. Abramson \\ ${ }^{I}$ Departments of Psychology and Zoology, Laboratory of Comparative Psychology and Behavioral Biology, \\ ${ }^{2}$ Department of Psychology, Laboratory of Child Behavior Research, \\ Oklahoma State University, 116 N. Murray, Stillwater, OK 74078, USA
}

\author{
Article history \\ Received: 15-06-2017 \\ Revised: 25-07-2017 \\ Accepted: 16-08-2017 \\ Corresponding Author: \\ Katherine E. Riley \\ Departments of Psychology \\ and Zoology, Laboratory of \\ Comparative Psychology and \\ Behavioral Biology, \\ Oklahoma State University, \\ 116 N. Murray, Stillwater, OK \\ 74078, USA \\ Email: keriley0@gmail.com
}

\begin{abstract}
The labyrinth was investigated as a means of generating stimuli in a locus of control paradigm. The experiment consisted of 56 undergraduates. Following completion of the Rotter I-E locus of control measure, participants negotiated a steel ball through a series of 44 obstacles over several trials. Analyses revealed evidence of greater persistence in participants with an internal locus of control than in participants with an external locus of control. The findings of this experiment indicate that the labyrinth is a useful apparatus to study locus of control under laboratory conditions.
\end{abstract}

Keywords: Locus of Control, Labyrinth, Persistence

\section{Introduction}

Over the years, there has been much debate in the literature concerning the reliability and validity of a psychological effect known as Locus Of Control (LOC) (Duttweiler, 1984). LOC is defined as the extent to which persons attribute the outcome of their behavior to either internal or external factors.Persons with internal locus of control attribute the outcomes of their behavior to either personal characteristics or internal factors and persons with external control attribute their behavior to external factors such as luck, fate, or powerful others (Rotter, 1990).

Since its discovery in 1954 (Rotter, 1954), LOC has been an active research area from 1954 with 2 articles to 7,422 articles by 2016. Despite the study of locus of control as a historically active area of research LOC studies have been in decline over the past decades (see Fig. 1). The decline in LOC studies is due to a distortion of the construct resulting from misuse and misinterpretations caused by ignoring the theoretical framework. Locus of control provided an outwardly simple explanation for interpreting behavior, but instead of building upon previous research, much of the literature that followed focused on individual differences (Rotter, 1990). Many studies conducted did not use operational definitions or clear measurements (Rotter, 1990). Therefore, studies on LOC have declined over the decades as psychologists manipulated LOC to fit their own research purposes.
We believe that one of the issues hindering a complete understanding of LOC is the lack of a reliable method to measure it. Typically, LOC is measured by a variety of survey instruments after the participant has engaged in some activity. For example, a study conducted on task performance administered questionnaires after participants engaged in a computerized task (Arnold, 1985). We think it is better to provide the survey before the task in order to help predict performance. In addition, recent studies have neglected to give a task, but merely use multiple questionnaires, including an LOC measure, to predict performance (Bodill and Roberts, 2013). Due to the psychometric issues regarding LOC measures, administering the survey prior to an experimental task can create a reliable method to study LOC. The results of recent studies could be improved by using an experimental method rather than relying on survey measures alone. There is much debate on the psychometric properties of LOC surveys as well as the creation of LOC surveys to test specific populations (e.g. health, academics and age) that create issues for future LOC research.

The purpose of this paper is to describe the use of the labyrinth to generate the psychological stimuli necessary to create locus of control. The wooden labyrinth is familiar to many people of a previous generation where the goal of the game is to negotiate a steel ball around a series of holes until the end point is reached. 


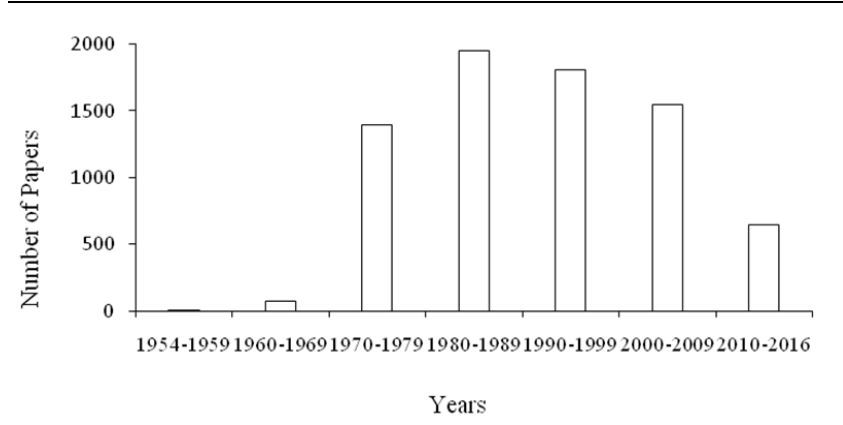

Fig. 1. The number of papers containing the subject heading "locus of control" for each decade from 1954 to 2016. Search conducted using the psycINFOdata base on the Search engine EBSCOhost

The user manipulates two knobs controlling the inner and outer boards, thus guiding the direction of the ball along a drawn path. The labyrinth can easily produce frustration especially in the novice user. In addition to producing frustration, the use of the labyrinth has much to recommend it for LOC studies. First, it is a readily available low cost device. It can be found on the internet and many toy stores for under $\$ 50.00$. Second, it is a standard device. Third, it allows the researcher to measure frustration directly either as defined by the number of holes negotiated or by adjunct behavior associated with frustration such as verbal comments, cleaning of the steel ball, etc. Fourth, the labyrinth can be used as a training tool to facilitate learning because it requires skill and utilizes trial-by-trial learning as well as manipulation of the inter-trial interval.

Participants can be trained to improve performance on the labyrinth over a certain period of trials, thus supporting the assertion that locus of control is not a fixed trait, but can be influenced by experience (Rotter, 1990). Finally, the researcher can measure persistence by total amount of time (in minutes) participants spend completing trials on the labyrinth. The labyrinth presents a skilled task that allows for a uniform situation in order for participants to adjust freely their expectancies for success or failure by previous performance on trials, thus generating the locus of control. We expected that participants with an internal locus of control would persist longer on the labyrinth than participants with an external locus of control.

\section{Method}

\section{Participants}

Participants were 57 undergraduate student staking introductory psychology classes. They were recruited through the (SONA system is an online study participation system used for psychological research) SONA system at Oklahoma State University and assigned research participation credit for their involvement in the experiment. One participant was removed from data analysis due to an incomplete survey; therefore, data analysis included 56 participants. The sample included slightly more female than male participants, with 33 females and 23 males. Participants were predominantly white $(71.4 \%)$ and freshman in college $(73.2 \%)$ with a mean age of 19.4 years $(\mathrm{SD}=1.34)$.

\section{Measures}

Participants filled out a demographic form asking for age, gender, ethnic background, marital status, grade point average, income, year in school, current frustration level and previous experience with mazes, labyrinths, or puzzles. Participants also rated their frustration level on a scale ranging from 0 to 100 in increments of 10 .

\section{Locus of Control}

Participants completed the Rotter 29-item InternalExternal Control Scale (I-E Scale) to indicate locus of control. The scale is a forced choice scale and ranges from 0 to 23 and includes 6 filler items to hide intent (Phares, 1976). A score of zero reflects an extreme perception of internal control while a score of 23 reflects an extremely external preference (Forte, 2005).

Each trial length was recorded in seconds with a stopwatch. The number of the hole the ball fell into was also recorded. Lastly, total time spent on the labyrinth in minutes was recorded. Time spent and number of trials completed served as two measures of persistence.

\section{Apparatus}

The apparatus used was the wooden labyrinth. It can be purchased online at http://www.shopgadgetsandgizmos.com/product/24968/ for the cost of $\$ 19.99$. The playing surface contains a path (black line) drawn through a course of 44 holes and obstacles. The labyrinth has two knobs, one on the front and the other on the right side. The front knob rotates the board left to right and the side knob rotates the board front to back. By using both knobs, participants were able to maneuver the board in a series of motions to guide the ball along the designated path from the start to finish line. Figure 2 provides a visual of the labyrinth used during the experiment.

\section{Procedure}

Participants entered the laboratory and completed the consent form, demographic form and the I-E Scale. The survey was administered prior to the experiment in order to help predict performance. After completing the forms, participants were given instructions on how to operate the labyrinth (e.g. knob placement and maneuverability) and how to complete the experiment. 


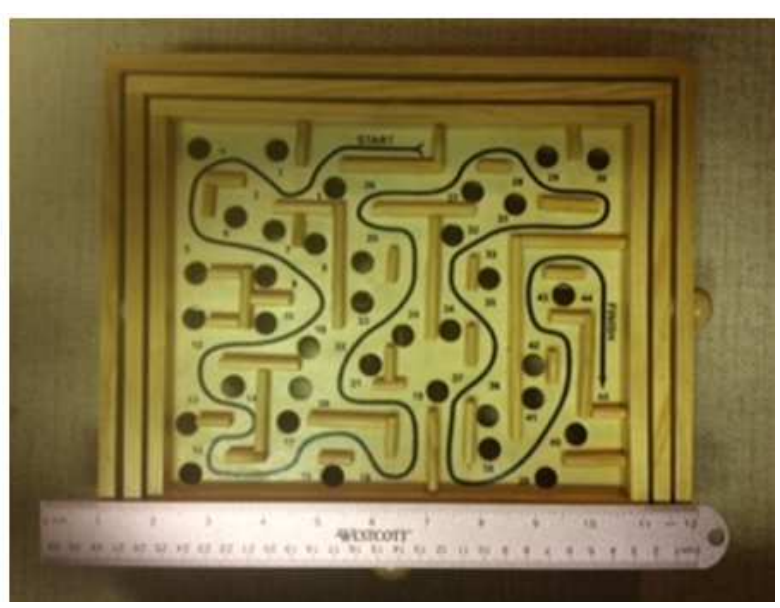

Fig. 2. The labyrinth device used for the experiment. It is 11 inches long, 10 inches wide and 3 inches high. A ruler is provided for an accurate depiction of the dimensions. Two knobs control the playing surface of the labyrinth and tilt the board in order to navigate the ball along the path. The labyrinth contains 44 holes and wooden obstacles

Participants were told their objective was to make it as far as they were able in the labyrinth by navigating the ball along the path for up to $25 \mathrm{~min}$. Participants were allowed to choose between two identical labyrinths, so that participants would not attribute failure to the apparatus prior to the experiment. No practice trials were given in order to predict how locus of control influenced persistence of completing more trials. Participants could stop when they chose, or continue to complete trials for the entire amount of time. Any questions were answered before beginning. If participants inquired about their performance relative to others, a standard response ("Everyone does about the same as you.") was given in order to avoid influencing participants' performance. The experiment ended when the participant indicated that he/she wanted to stop or at $25 \mathrm{~min}$. After the experiment, participants again reported their frustration and total time was recorded.

This experiment utilized self-paced inter-trial intervals and a variable number of trials (up to $25 \mathrm{~min}$ ) to measure persistence and correlate it to participants' locus of control scores. Participants were allowed to control the inter-trial interval in order to feel in control and perform naturally without feeling influenced by the researcher. The participant determined the number of trials within the $25 \mathrm{~min}$ time period. Participants were given the same instructions and completed the experiment under the same conditions.

\section{Results}

Participant's I-E Scale scores (I-E) included a minimum of 8 and a maximum of 18 , with the mean $(M$ $=11.64, S D=2.15$ ). A score from 0 to 11 is considered more internal while $12-23$ is considered more external
(Forte, 2005). Overall, participants represented a wide range of scores with $44.6 \%$ representing a more internal locus of control and 55\% representing a more external locus of control. However, 24 out of 56 participants were clustered in the middle with scores at 12 and 13 . Figure 3 shows the frequency distribution of LOC scores.

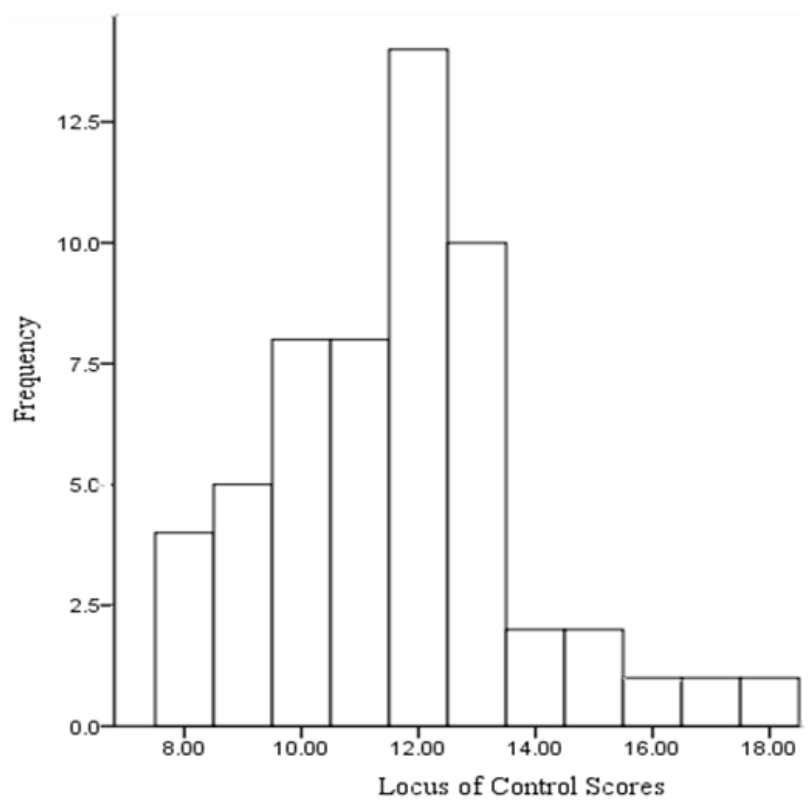

Fig. 3. Frequency distribution for participant's locus of control scores. A lower score indicates a greater internal LOC and a higher score indicates a greater external LOC

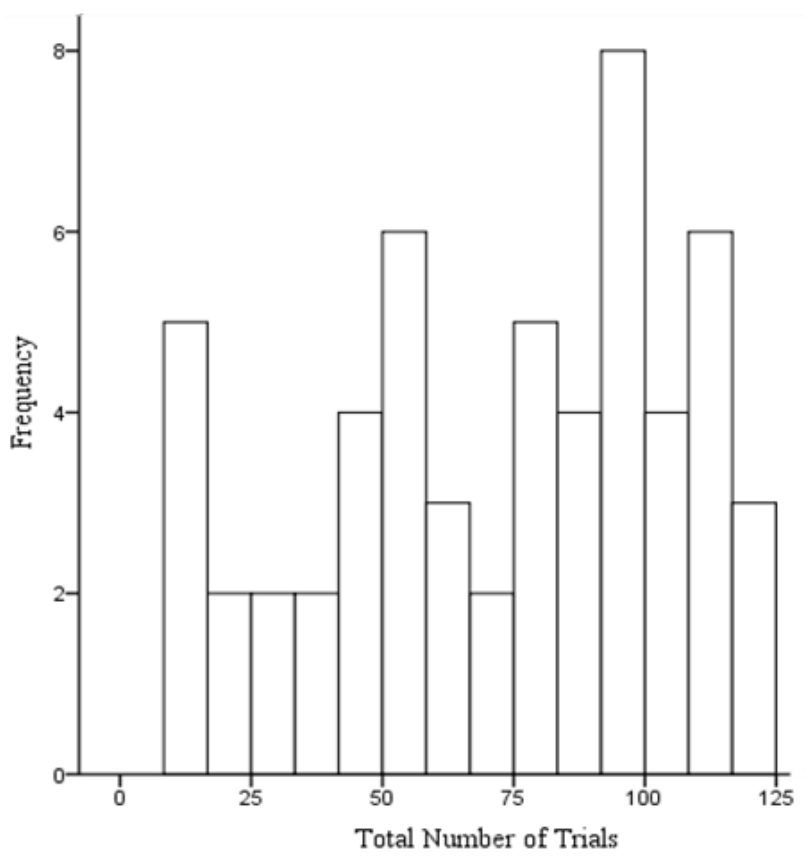

Fig. 4. Frequency distribution for number of trials completed by participants in a $25 \mathrm{~min}$ time period 
Table 1. Correlations for Locus of Control Scores and Persistence on the Labyrinth

\begin{tabular}{lllll}
\hline & & Total Time in Minutes & Total Number of Trials & Total I-E Scale Scores \\
\hline Total Number of & Pearson Correlation & $0.893^{* *}$ & -- & -- \\
Trials & Sig. (2-tailed) & 0.000 & $* * .310^{*}$ & -- \\
Total I-E Scale Scores & & $0.346^{* *}$ & 0.020 & - \\
& & 0.009 & 0.00 & \\
\hline
\end{tabular}

Notes: $\mathrm{N}=56$

**. Correlation is significant at the 0.01 level (2-tailed)

*. Correlation is significant at the 0.05 level (2-tailed)

Table 2. Correlations for demographics

\begin{tabular}{llllll}
\hline & & $\begin{array}{l}\text { Total I-E } \\
\text { Scale Scores }\end{array}$ & $\begin{array}{l}\text { Gender of } \\
\text { Participant }\end{array}$ & $\begin{array}{l}\text { Age of } \\
\text { Participant }\end{array}$ & GPA \\
\hline Gender of Participant & Pearson correlation & $-.327^{*}$ & -- & - & -- \\
& Sig. (2-tailed) & 0.014 & & - & - \\
Age of Participant & & 0.198 & -0.065 & - & - \\
GPA & & 0.143 & 0.635 & -0.215 & - \\
& & -0.008 & 0.033 & 0.129 & \\
\hline
\end{tabular}

Notes: $\mathrm{N}=56$

GPA: $\mathrm{N}=51$

*. Correlation is significant at the 0.05 level (2-tailed).

Table 3. Correlations for Previous Experience

\begin{tabular}{lllllll}
\hline & & $\begin{array}{l}\text { Electronic } \\
\text { Puzzles }\end{array}$ & $\begin{array}{l}\text { Touch } \\
\text { Puzzles }\end{array}$ & $\begin{array}{l}\text { I-E Scale } \\
\text { Scores }\end{array}$ & $\begin{array}{l}\text { Total Time } \\
\text { (min) }\end{array}$ & $\begin{array}{l}\text { Total Number } \\
\text { of Trials }\end{array}$ \\
\hline Electronic puzzles & Pearson correlation & 1 & $0.589^{* *}$ & 0.048 & -0.110 & -0.105 \\
Touch Puzzles & Sig. (2-tailed) & & 0.000 .727 & & 0.420 & 0.443 \\
& & -- & -- & 0.015 & 0.045 & 0.013 \\
& & & & 0.914 & 0.741 & 0.924 \\
\hline
\end{tabular}

Notes: $\mathrm{N}=56$

**. Correlation is significant at the 0.01 level (2-tailed)

*. Correlation is significant at the 0.05 level (2-tailed)

Table 4. Correlations for Frustration After, Locus of Control and Persistence

\begin{tabular}{llllll}
\hline & & Frustration & I-E Scale & Total Time & Total Number \\
\hline \multirow{3}{*}{ Frustration after } & & After & Scores & (Min0 & of Trials \\
& Pearson correlation & 1 & -0.053 & $-0.315^{*}$ & $-0.395^{* *}$ \\
Locus of Control Scores & Sig. (2-tailed) & & .699 & 0.018 & 0.003 \\
& & -- & -- & $0.346^{* *}$ & $0.310^{*}$ \\
& & & & 0.009 & 0.020 \\
\hline
\end{tabular}

Notes: $\mathrm{N}=56$

*. Correlation is significant at the 0.05 level (2-tailed).

**. Correlation is significant at the 0.01 level (2-tailed).

Measures of persistence included total time in minutes and total number of trials. Participants completed an average of 79 trials $(S D=33.36)$ in an average of $21 \min (S D=7.75)$. The minimum number of trials completed was 9 and the maximum number of trials completed was 120 . Twenty-six participants completed trials for the maximum amount of time $(25 \mathrm{~min})$ while 9 gave up in under ten minutes. Figures 4 and 5 show frequencies of the total amount of trials completed and the amount of time spent completing trials on the labyrinth.

We found evidence that LOC is associated with persistence on the labyrinth. To find evidence of persistence, locus of control scores were correlated with the amount of time spent on the labyrinth and the number of trials completed within the 25 min time period. Correlations revealed that I-E Scale scores were significantly positively correlated with levels of persistence. As I-E Scale scores increased, number of trials and time spent with the labyrinth also increased. Participants with higher locus of control scores completed more trials and spent more time with the labyrinth. Table 1 shows the association between I-E scores and persistence.

In order to control for the effects of demographic variables such as age, gender and GPA, these were correlated with I-E Scale scores and results are presented in Table 2. 


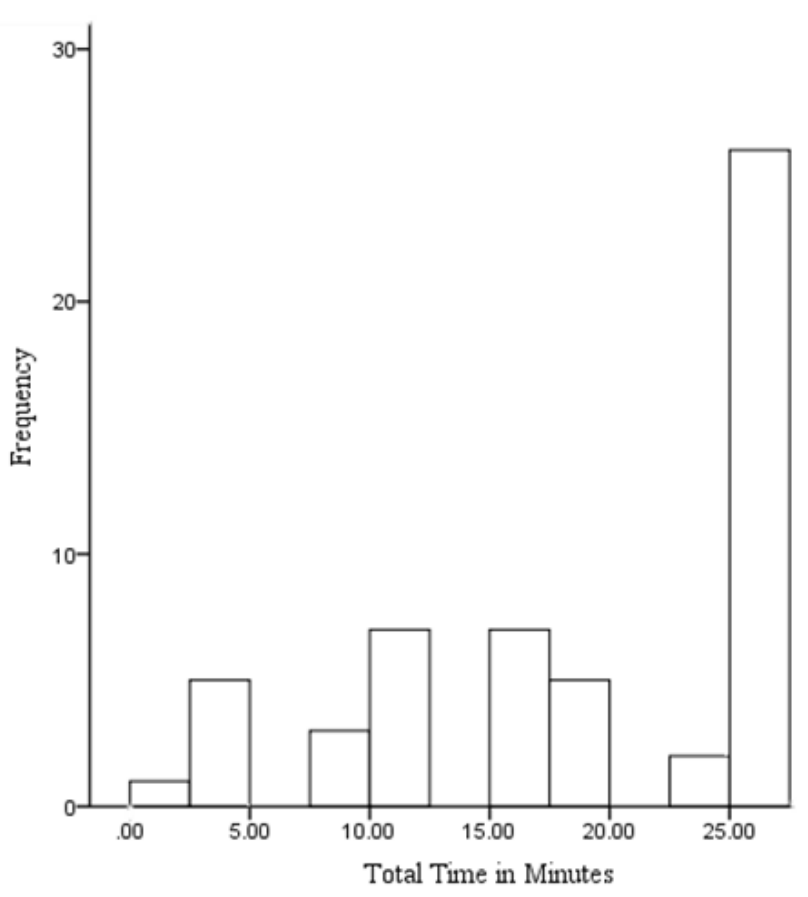

Fig. 5. Frequency distribution for the total time in minutes participant's spend completing trials on the labyrinth

Yearly income and ethnicity were examined; however, both of these variables were homogenous and did not have a significant impact on the data. Locus of control was not significantly correlated with age, gender and GPA. However, an independent samples t-test for gender differences and locus of control revealed that males had a slightly higher locus of control than females. There was a significant difference in the gender of participant $(M=12.47, S D=2.15)$ and the locus of control scores $(M=11.06, S D=1.98) ; t(54)=2.54, p=0.014$. Gender differences were specifically examined because research suggests that societal pressures and socialization affects males and females differently when tasks involve success and performance (Phares, 1976).

Previous experience was assessed in order to determine if it would influence persistence on the labyrinth. Correlations are presented in Table 3.38\% of participants indicated they rarely used electronic puzzles and $59 \%$ indicated they rarely used puzzles that can be physically touched. Only one participant indicated use of electronic puzzles "very often," and 11\% reported sometimes using touch puzzles. Overall, previous experience did not influence persistence on the labyrinth.

Frustration measures were taken and $68 \%$ of participants indicated no frustration at all prior to starting the experiment. After the experiment, most participants indicated some degree of frustration with an average rating of 40 on a scale ranging from 0 to 100 . I-E Scale scores and frustration ratings were significantly negatively correlated, indicating that those with a more internal locus of control were less likely to be frustrated. Correlations are presented in Table 4.

\section{Discussion}

We found a significant association between persistencein completing more trials and more internal LOC. This finding supports previous research on behavioral characteristics of both internals and externals (Phares, 1976). Internals are positively reinforced for their successes and perceive themselves as in control of preventing aversive stimuli. They are motivated to perform better after achievement and downplay failures. Externals are less motivated by their successes because success is attributed to outside elements, so they are more likely to quit (Phares, 1976). This finding is important because it provides support that the labyrinth provides the necessary conditions to create LOC. The labyrinth allows participants to make attributions for successes and failures and learning and performance is guided by the participant's internal or external control of reinforcement.

Social Learning Theory suggests the locus of control does not operate independently but within a theoretical context and is a model for explaining behavior based on available choices. It is used to help predict behavior based on three contingencies, expectancy of reinforcement, reinforcement value and the situation (Phares, 1976). Expectancy is a subjective probability that a behavior will be reinforced in a situation. Expectancies can be learned based on past experiences and familiar situations. Reinforcement is anything that influences behavior and the value placed on reinforcement is the most preferred type of reinforcement if other options are unavailable. Finally, the psychological situation influences behavior and is important for predicting behavior in conjunction with the first two variables (Phares, 1976). By considering these concepts, an individual's expectancy can generalize to other situations and yields a preference for either an internal or external belief system. This aids the individual to make attributions for success and failure in social situations. Persons with an internal belief expect the results of their behavior to either come from personal characteristics or internal control and persons with an external belief attribute their behavior to outside factors such as luck, fate, or powerful others (Rotter, 1990).

When first defining the construct, the I-E variable referred to the degree a person willingly accepted personal responsibility for outcomes (Lefcourt, 1966). Internals enjoy manipulating their environment, taking advantage of opportunities and influencing outcomes. They have a greater sense of control, which makes it more probable that they will seek out beneficial information (Phares, 1976). Externals do not feel that they have control over their environment and lack of 
control results in lower performance, trouble coping and increased anxiety (Phares, 1976). They do not perceive their success as an aspect of themselves, so they are not positivity reinforced. Situational cues such as automatic changes and emotions can negatively affect behavior because it worsens learning and performance. However, internals are positively reinforced for their successes and perceive themselves as in control of preventing aversive stimuli. They are motivated to perform better after achievement and downplay failures. Externals are less motivated by their successes because success is attributed to outside elements, so they are more likely to quit (Phares, 1976).

The use of the labyrinth in order to implement applied psychological concepts into formulating experiments helps expands upon previous learning research that views the construct as a changeable personality characteristic instead of a distinct individual difference. This is important in order to bring clarity back into interpreting the construct and for advancing sound knowledge (Rotter, 1990). The labyrinth is an ideal apparatus to generate experimentally locus of control in a laboratory setting because it produces real data and quantifiable results that can be correlated with LOC measures to strengthen construct validity. It can be used to design instrumental learning experiments and to formulate operation definitions for variables studied (Baskin et al., 2013). This is important because many studies conducted did not use operational definitions or clear measurements (Rotter, 1990). Psychologists at the time were not considering learning principles that were essential to LOC. Some of the misconceptions of LOC include issues with classifying and categorizing internals versus externals. The value of reinforcement was not considered; rather a unidimensional approach took place (Kormanik and Rocco, 2009). Participant's expectancy of reinforcement, either internal or external LOC, will influence persistence and serve as the reinforcement for choosing to complete more trials.

The labyrinth provides a solution to some of the previous issues discussed in the literature on locus of control (Rotter, 1990). Between the 1960s through 1990s, studies on locus of control spiked, however several factors contributed to the problems occurring in studies on the construct. First, interest in finding explanations to the causes of human behavior, led to misinterpretation of the construct. Locus of control provided an outwardly simple explanation for interpreting behavior, but instead of building upon previous research, much of the literature that followed focused on individual differences (Rotter, 1990). Second, the cognitive revolution shifted psychological thinking from a primary behavioral perspective to a cognitive perspective (Abramson, 2013). The outcome resulted in researchers not fully considering the theoretical framework needed to understand the concept, which led to misinterpretations, biases and distorted views on locus of control. Essentially, psychologists adopted the view of locus of control as a fixed personality trait, rather than one that can change through experience (Kormanik and Rocco, 2009). The labyrinth uses trial-by-trial learning, thus participants can be trained to improve their performance over time and shift the locus of control.

The current study provides practical implications to anyone interested in researching locus of control. Questionnaires alone do not provide enough information to understand entirely how the locus of control influences behavior. By utilizing the labyrinth as an experimental measure, researchers can obtain more information from a quick task to predict behavior in a practical situation in addition to the classroom and laboratory settings. The labyrinth task can measure a wide variety of variables such as mood, emotional states and compliance behaviors, which gives it a practical application for future research in locus of control studies. Researchers can use survey instruments first and then provide participants with the labyrinth task. The locus of control measure is enhanced by giving participants a task that requires persistence and elicits frustration. The researcher will have more information in which to interpret the results of the study beyond standard survey measures.

The implications of this study can advance current knowledge of the locus of control construct by providing researchers with a method to help them predict outcomes to specific situations. Current locus of control literature aims to predict outcomes for the populations under study. For example, a study testing a parenting intervention uses survey measures for a parent's locus of control and stress level in order to predict disruptive behaviors in children (Moreland et al., 2016). By using the labyrinth, researchers can obtain greater information about their predictions. Current research also investigates the links between locus of control, depression and coping strategies used by cancer patients (Aarts et al., 2015), as well as how these patients use and gather information about their illness in relation to their locus of control (Keinki et al., 2016). Utilizing the labyrinth as part of the methodology of future studies can benefit researchers by producing results that are more reliable.

Sample size and demographics may have caused some limitation to the study. First, our sample consisted of primarily white college freshman. The results will not likely generalize to a wider population. There was no significant difference in GPA and LOC scores, which is most likely due to the sample being predominantly similar.

We believe this study will provide a start for more labyrinth studies in the future. There is a surfeit of reasons providing support for the labyrinth as a learning tool, as well as theoretical support for the labyrinth in LOC studies (Baskin et al., 2013). 


\section{Conclusion}

We believe this study will provide a start for more labyrinth studies in the future. There is a surfeit of reasons providing support for the labyrinth as a learning tool, as well as theoretical support for the labyrinth in LOC studies (Baskin et al., 2013).

\section{Acknowledgement}

I would like to thank my professors, Dr. Abramson and Dr. Sullivan for giving me the opportunity to collaborate on this research. Thank you both for your mentorship and support.

\section{Authors' Contributions}

Katherine E. Riley: Conducted the investigation and collected data, assisted in data interpretation and analysis, drafted the original manuscript and final versions and edited the manuscript.

Maureen A. Sullivan: Performed data analysis and interpretation, provided laboratory space, supervised and validated the research activities and reviewed and edited the manuscript.

Charles I. Abramson: Designed and formulated the study's aims, provided the labyrinths used in the experiment, supervised and validated the research activities and reviewed and edited the manuscript.

\section{Ethics}

There are no conflicts of interest associated with this research.

\section{References}

Aarts, J.F., L. Deckx, D.L. Abbema, V.G. Tjan-Heijnen and M. Akker et al., 2015. The relation between depression, coping and health locus of control: Differences between older and younger patients, with and without cancer. Psycho-Oncology, 24: 950-957. DOI: $10.1002 /$ pon.3748

Abramson, C.I., 2013. Problems teaching the behaviorist perspective in the cognitive revolution. Behavioral Sci., 3: 55-71. DOI: 10.3390/bs3010055

Arnold, H.J., 1985. Task performance, perceived competence and attributed causes of performance as determinants of intrinsic motivation. Academy Management J., 28: 876-888. DOI: 10.2307/256242
Baskin, K.E., C.C. Cushing and C.I. Abramson, 2013. Using the labyrinth as a teaching tool in psychology. Innovative Teaching, 2: 10. DOI: $10.2466 / 07.08 . I T .2 .10$

Bodill, K. and L.D. Roberts, 2013. Implicit theories of intelligence and academic locus of control as predictors of studying behavior. Learning Individual Differences, 27: 163-166. DOI: 10.1016/j.lindif.2013.08.001

Duttweiler, P.C., 1984. The internal control index: A newly developed measure of locus of control. Educ. Psychol. Measurement, 44: 209-221. DOI: $10.177 / 0013164484442004$

Forte, A., 2005. Locus of control and the moral reasoning of managers. J. Bus. Ethics, 58: 65-77. DOI: $10.1007 / \mathrm{s} 10551-005-1387-6$

Keinki, C., E. Seilacher, M. Ebel, D. Ruetters and I. Kessler et al., 2016. Information needs of cancer patients and perception of impact of the disease, of self-efficacy and locus of control. J. Cancer Educ., 31: 610-616. DOI: 10.1007/s13187-015-0860-x

Kormanik, M.B. and T.S. Rocco, 2009. Internal versus external control of reinforcement: A review of the locus of control construct. Human Resource Development Rev., 8: 463-483. DOI: $10.1177 / 1534484309342080$

Lefcourt, H.M., 1966. Internal versus external control of reinforcement: A review. Psychol. Bulletin, 65: 206220. DOI: $10.1037 / \mathrm{h} 0023116$

Moreland, A.D., J.W. Felton, R.F. Hanson, C. Jackson and J.E. Dumas, 2016. The relation between parenting stress, locus of control and child outcomes: Predictors of change in a parenting intervention. J. Child Family Studies, 25: 2046-2054 DOI: $10.1007 / \mathrm{s} 10826-016-0370-4$

Phares, J.E., 1976. Locus of Control in Personality. New Jersey: General Learning Press.

Rotter, J.B., 1954. Social learning and clinical psychology. New York: Prentice-Hall.

Rotter, J.B., 1990. Internal versus external control of reinforcement: A case history of a variable. Am. Psychol., 45: 489-493.

DOI: 10.1037/0003-066X.45.4.489 